\title{
Proposal to Develop Techniques Using Magneto-Optic and Electro-Optic Effects in Optical Fiber for CTR Diagnostics
}

G. I. Chandler

F. C. Jahoda

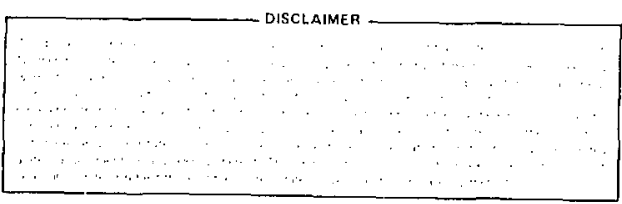


PROPOSAL TO DEVELOP TECHNIQUES USING MAGNETO-OPTIC

AND ELECTRO-OPTIC EFFECTS IN OPTICAL FIBER FOR CTR DIAGNOSTICS

by

G. I. Chandler and F. C. Jahoda

\begin{abstract}
We discuss the developing technology of measuring electric and magnetic fields with optical fibers using the Faraday and Kerr effects, magnetostriction, and Sagnac interferometry. We review the measurement of induced birefringence in the presence of natural birefringence. We propose the use of these effects in making measurements in the fusion research program, with $2 \mathrm{~T}-40$ as an example.
\end{abstract}

\title{
I. INTRODUCTION
}

This report addresses the rationale and feasibility of using the magneto-optic (Faraday effect) and electro-optic (Kerr effect) properties of fused silica single-mode optical fibers to measure magnetic and electric fields in controlled thermonuclear reaction (CTR) experiments.

Since the first low-1oss $(\sim 20 \mathrm{~dB} / \mathrm{km})$ fibers were created by Corning in 1969, the optical fiber industry has grown dramatically as applications in the communications field have been realized with great success. CTR-Division of Los Alamos National Laboratory has developed this technology to solve problems wth electromagnetic interference in data communications, trigger pulse timing, and diagnostics.

Researchers studying the optical properties of comunication fibers were Inevitably led to the measurement of polarization parameters and the unsurprising discovery of birefringence and photoelasticity. 1 The possibility of doubling the channel-carcying capacity of a single-mode fiber by using both orthogonally polarized modes led to efforts to produce fibers in which the coupling between modes is minimized (so-called polarization-maintaining fibers), and the Kerr and Faraday effects ${ }^{2}$ were used to make measurements on candidate fibers. 
Electric power companies used fiber optics very early for isolation when coupling current and voltage measurements down from high-voltage lines. They had also used the Faraday effect to measure line currents, and the idea of using the Faraday effect in fibers to produce a simple, accurate, electrically isolated current measurement is the most important driving force behind efforts to produce very low linear polarization birefringence optical fibers. The British ${ }^{3}$ and Germans ${ }^{4-6}$ are far advanced in these areas. An excellent review article was written by Smith. 7

The Navy has commissioned the Naval Research Laboratories (NRL) to study the entfre field of fiber optics sensors, and a great deal of work has been accomplished, with primary emphasis on magnetic field detection and urderwater hydrophones. A "Technology Assessment" is produced by NRL at irregular intervals, ${ }^{8}$ and annual workshops are held.

\section{PROPERTIES OF FIBERS}

An optical fiber can be treated as a dielectric wave-guide and has a specific number of propagating modes dependent on the vaiue of the normalized frequency $v$ :

$$
v=\frac{\pi d}{\lambda}\left(n_{1}^{2}-n_{2}^{2}\right)^{1 / 2} \text {, }
$$

where $d=$ diameter of core,

$$
\begin{aligned}
& \mathrm{n}_{1}=\text { Index of core, } \\
& \mathrm{n}_{2}=\text { Index of cladding, and } \\
& \lambda=\text { wavelength. }
\end{aligned}
$$

$\mathrm{NA}=\left(\mathrm{n}_{1}^{2}-\mathrm{n}_{2}^{2}\right)^{1 / 2}=$ numerical aperture, the sine of the half-angle of the fiber acceptance cone.

Table $I^{9}$ lists the number of propagating modes for various ranges of $v$, the maximum core diameter that applies for 633-nm 1ight, and a (typical) NA of 0.10. Single-mode fibers (really two modes of orthogonal polarization) are commerclally available and widely used for high-bandwidth communications 
TABLE I

PROPOGATTNG MODES FOR VARIOUS RANGFS OF $v$

v No. of Modes $\quad$ d Max (Im)

\begin{tabular}{|c|c|c|c|}
\hline & -2.4048 & 2 & 4.84 \\
\hline 2.4048 & -3.8317 & 6 & 7.72 \\
\hline 3.8317 & -5.1356 & 12 & 10.36 \\
\hline 5.1356 & -5.5201 & 16 & 11.12 \\
\hline 5.5201 & -6.3802 & 20 & 12.86 \\
\hline
\end{tabular}

links. A typical single-role fiber has a 4-im core diameter, 80-um cladding diameter, and a silicone rubber jacket for an overall outside diameter of 500 1⿴囗十 *

Early measurements ${ }^{l}$ revealed that fibers have falrly large intrinsic linear birefringence that is reasonably uniform along their lengths. Additional linear birefringence is induced by bending and clamping stress. Measured values of linear birefringence (expressed in degrees of relative phase retardation per centimeter) are listed in Table II for various fibers as reported in the literature. Some of them are produced in such a way as to deliberately maximize or minimize this parameter. Circular birefringence can be introduced by twisting the fiber, and may be present in the relaxed fiber as well.

The linear birefringence of the fiber may be related by stress-optic theorylo to the ellipticity of the core, and fibers with very high birefringence have been produced by deliberately deforming the core.11 Those who work in this area typicaliy specify the "beat length," the distance for the retardation to change by a factor of $2 \pi$, and that distance has been reduced to $<1 \mathrm{~cm}$. This is one way to produce a polarization-maintaining fiber and is favored by American workers at Corning, ITT, and Bell Labs.

\footnotetext{
*ITT Type 1601 single-mode fiber. ITT Electro-Optical Products Division, 7635 Plantation Road, Roanoke, VA 24019
} 
TABLE II

REPORTED VALUES OF LINEAR BIREFRINGENCE

\begin{tabular}{|c|c|c|c|}
\hline Fiber Description & deg $\mathrm{cm}^{-1}$ & Date & Investigators \\
\hline Single-mode, $3.8-\mu \mathrm{m}$ core & 1.1 & 1972 & Kapron et al.1 \\
\hline Graded index, $50-\mu m$ core & 58 & 1975 & Papp and Harms ${ }^{6}$ \\
\hline Liquid core, 94- 4 m core & 0 & 1975 & Aulich et al. 5 \\
\hline $126-\mu \mathrm{m}$ core, $\mathrm{N}_{\mathrm{a}}-\mathrm{S}_{1} \mathrm{O}^{2}-\mathrm{B}_{2} \mathrm{O}_{3}$ & 0.7 & 1978 & Aulich et al. ${ }^{5}$ \\
\hline l10-ım core silica & 0.06 & 1978 & Aulich et al. 5 \\
\hline $5-\mu m$ core silica CVD & 0.06 & 1978 & Aulich et al. 5 \\
\hline 4.7-lan core silica & 66 & 1976 & Ramaswamy et al.10 \\
\hline $2-\mu \mathrm{m} / 3-\mu \mathrm{m}$ ell1ptical core & $277-72$ & 1979 & Ramaswamy et al. 11 \\
\hline 5-jun core sillca CVD & 0.1 & 1978 & Schneider et a1. 13 \\
\hline $7-1 m$ core & .25 & 1978 & Smith 19 \\
\hline 9-um core silica SVI & .022 & 1979 & Norman et a 1.3 \\
\hline
\end{tabular}

The European approach is to produce very round, stress-free $f$ ibers of very low linear birefringence. This is done at siemens $A G^{13}$ and the University of Southampton. 3

There are multimode fibers listed in Table II with rather low birefringence. These are not generally sultable for making polarization preserving devices because the light is depolarized rather quickly, apparently because of strong coupling between modes. Single-mode fibers maintain the degree of polarization very we11. We note a distinction here between the degree and the state of polarization (SOP); the latter changes because of birefringence, but that change need have no effect on the former. It is possible to launch a single mode in a multimode fiber, but it is difficult, and the geometry of most practical fiber devices will quickly cause the energy to spread to the other modes. For these reasons most Elber sensor work is done with single-mode 'f tbers.

The Faraday effect is the consequence of circular birefringence caused by a longitudinal magnetic field. The phenomenon of circular birefringence causes a rotation of the plane of linearly polarized 11ght. Equivalently, in terms of clrcularly polarlzed basts vectors of opposite sense, it is a phase retardation between those vectors. The rotation angle of linear polarization is glven in general by 


$$
F=V \int \vec{H} \cdot d \vec{l}
$$

or

$$
\mathrm{F}=\mathrm{V} \oint \overrightarrow{\mathrm{H}} \cdot \mathrm{d} \vec{l}=\mathrm{VI}
$$

for closed loops linking the current $I$. The Verdet constant $V$ is expressed in $\mathrm{rad} \mathrm{amp}^{-1}$. For silica at $633 \mathrm{~nm}, \mathrm{~V}=4.68 \times 10^{-6} \mathrm{rad} \mathrm{A}^{-1}$ (See Ref. 7).

The electro-optic Kerr effect is the production of linear birefringence with a transverse electric field, and the phase retardation is given by

$$
B=\pi \ell B_{K} E^{2},
$$

$$
\text { where } \begin{aligned}
E & =\text { transverse field strength, volt } \mathrm{cm}^{-1}, \\
\ell & =\text { exposed length, cro, and } \\
B_{K} & =\text { Kerr constant, measured }{ }^{2} \text { in a silica fiber as } 9 \times 10^{-15} \mathrm{~cm} \mathrm{v}^{-2} \text {. }
\end{aligned}
$$

III. POLARIZATION MEASUREMENTS

The state of polarization of light traveling along a flber evolves according to the characteristics of the fiber. Any fiber has orthogonal fast and slow axes (1inear birefringence) that rotate along the length of the fiber. If one thinks of the SOP at any point along the flber as the ellipse traced by the end of the electric fleld vector, that ellipse is characterized by an angle with respect to the local fast fiber axis (azimuth)(see Fig. 1). The ellipticity and azimuth evolve with the local values of the fast and slow axis Indexes of refraction, or the spectfic linear polarization phase retardations, and the azimuth alone evolves with the local circular birefringence (generated by shear stress caused by twisting or by magnetic fields). One can see that when both types of birefringence are present, the evolution of the SOP is not simple--it may be visualized to some extent in the 
so-called Poincaré sphere representation. (See Ref. 2 and a book on polarized light such as Shurcliff, 14 Swindell, 15 or Azzam and Bashara. ${ }^{16}$ )

Nevertheless, it is posstble to measure circular birefringence in the presence of linear birefringence. In F1g. 2 we see a conceptualization of a measurement system. Linearly polarized light is incident on the end of a fiber with its plane of polarlzation parallel to one of the fiber axes. The output beam is split into orthogonally polarized beams, the $\mathrm{r}$ axes at angles of $\pi / 4$ with respect to the fast and slow axes of the fiber at that end. We describe the procedure for location of the fast and slow axes of the fiber in the appendix. The Intensity $I_{1}, I_{2}$ of each beam is measured with a phorodiode, and the intensity-normalized quantity $T$,

$$
T=\frac{I_{1}-I_{2}}{I_{1}+I_{2}}
$$

is calculated with analog electronics 17,18 or, as we propose, by computer with analog-digital converters for data acquisition.

According to smith ${ }^{19} \mathrm{~T}$ is related to the several birefringence and optical parameters by

$$
T=-2 \cos \left(\delta_{2}+n\right) \sin x \sin \frac{\phi}{2}\left(\cos ^{2} \frac{\phi}{2}+\cos ^{2} \times \sin ^{2} \frac{\phi}{2}\right)^{1 / 2}
$$

The parameters in Eq. (6) are defined by reference to Figs. 3 and 4, in terms of $F$ and $\delta_{1}$. In Fig. 3, the fiber is shown In three parts: an Initial leading section of length $\ell_{0}$ between the input optics and the field-measurement region having total phase retardation of $\delta_{0}=l_{0} \delta_{s}$, where $\delta_{s}$ is the linear retardation per unit length of the fijer; the fleld-measurement region of length $\ell_{1}$, of total retardation $\delta_{1}$ and Faraday rotation angle F; and third, another field-free "lead" region connecting to the output opties with length $\ell_{2}$ and retardation $\delta_{2}$. The parameters $x$ and $\phi$ are defined by the trigonometric relationships of the right triangle shown in F1g. 4; and finally, 


$$
\eta=\tan ^{-1}\left(\cos x \tan \frac{\phi}{2}\right)
$$

Note that $\delta_{0}$ does not appear.

Equation (6) could be used directly with a computer to find $F$ and so determine the magnetic fleld or current because all other parameters are previously determined. However, the study of three limiting cases of Eq. (6) is instructive and helpful in laboratory work.

Case $1: F \gg \delta_{1} / 2, \delta_{2}$ (low linear birefringence).

$\because$ this 1 imit $\sin x+1, \cos x+\delta_{1} / 2 F, \phi / 2+F, \sin \phi / 2+\sin F, \cos$ $\phi / 2+\cos \mathrm{F}$, and $\cos \left(\delta_{2}+\eta\right)+1$. Substituting into Eq. (6) and using a double-angle trig identity, we get simply

$$
\mathrm{T} \simeq-\sin 2 \mathrm{~F}
$$

Case 2: $\mathrm{F} \ll \delta_{1} / 2, \delta_{2} \ll \delta_{1}$.

Again using Fig. $4, \phi+\delta_{1}$, sin $x \rightarrow 2 F / \delta_{1}, \cos x=\delta_{1} / \phi \rightarrow 1, \sin$ $\phi / 2+\sin \delta_{1} / 2, \cos \phi / 2+\cos \delta_{1} / 2, \tan \phi / 2 \rightarrow \tan \delta_{1} / 2$ so that $\cos$ $\left(\delta_{2}+\eta\right)+\cos \delta_{1} / 2$. Substituting into Eq. (6) and using the double angle Identity we get

$$
T \simeq-2 \mathrm{~F} \frac{\sin \delta_{1}}{\delta_{1}}
$$

A better approximation for Case 2 with values of $\delta_{1}$ and $F$ in the same range is suggested by Smith in Ref. 7:

$$
\mathrm{T} \simeq 2 \mathrm{~F} \frac{\sin \phi}{\phi}
$$

Case 3: A clever approach 20,21 allows normal fibers of moderate values of $\delta_{s}$ to be used by biasing $F$ with circular birefringence induced by twisting the fiber. Then $F$ is replaced by $\alpha+F$, where $\alpha=g \tau$; $g$ is a constant relating the induced circular birefringence $\alpha$ to the twist per unit length $\tau$, and has values in the range $0.1-0.16$. We get $\mathrm{F}+\alpha \gg \delta_{1} / 2, \delta_{2}$, with the result the same as in Case 1 if we set $\alpha=\mathrm{n} \pi$, with $\delta_{2}=\mathrm{m} \pi / 2$ and $\mathrm{n}$ and $m$ integers, and then 


$$
T \simeq-\sin 2 F
$$

Note that Case 1 gives the justfflcatlun for making very low linear birefringence fibers for current sensing, whereas Case 2 argues for $h 1, h$ In practical advantanges and limftations of all three cases.

Temperature can be a problem in a highly stressed fiber because of the Interaction between coefficients of expansion, the stress-optic coefficients, and birefringence. The high birefringence fibers (Case 2) use severely deformed cores to achieve that property and hence may be expected to show considerable temperature sensitivity. The same is true of fibers twisted as In Case 3. Ciearly, the most desirable fiber would appear to be the low Inear birefringence type that is not available in the US.

We compared values calculated from Eqs. (6) and (8) for $2 \mathrm{~T}-40$ conditions and a single-turn loop of silfca fiber of very low birefringence ( $2 \mathrm{deg} \mathrm{m}^{-1}$ ). inis includes a $1.5-\mathrm{m}$ loop length and $2-\mathrm{m}$ leads. The values obtained agreed to about $1 \%$. Equation (6) is plotted in Fig. 5 for the parameters discussed. The perk occurred near $180 \mathrm{kA}$, so that to make a Faraday optical loop for $2 T-40$, we would need some means of detecting which side of the peak or the following zero one was on (similar to counting whole fringes in an interferometer), or we could use a different number of turns. At $400 \mathrm{kA}$ the Faraday rotation for one turn would amount to about 107 degrees. 
In Fig. 6 we convolve the transfer function of Fig. 5 with a $2 T-40 I_{\phi}$ waveform for two different values of peak current, $195 \mathrm{kA}$ and $390 \mathrm{kA}$. The sinusoidal nature of the polarimeter output causes wide variation of sensftivity depending on current level. Obviously it is best to use this technique on the steep part of the response curve, praferably on the first cycle; that is, with current less than around $100 \mathrm{kA}$. This technique is best used to look for "fine structure" in high current levels, however, by biasing the device to get to a steep part of the curve. The expected resolution is around $1 \%$, or 1000 amps, for a one-turn loop of fiber and carefully adjusted analog electronics.

\section{SAGNAC INTERFEROMETER}

Arditty reported 22 at the Integrated optics and optical Communications Conference this year in San Francisco (IOOC' 81 ) that the Faraday effect was measured by using optical fiber in a Sagnac interferometer. This is a technique widely discussed for use in rotation sensing (optical gyrosopes) and promises to be simpler and more sensitive than the direct optical polarimetric method just described.

Figure 7 illustrates the concept. The 11nearly polarized output of a laser passes through a beam splitter and a circular polarizer and is incident on both ends of a fiber optic loop. The output is circularly polarized (only one polarization is needed) so that the light traveling in both directions has the same (say left-hand) polarization. The component of magnetic field along the fiber modifies the indexes of refraction for left-handed polarization differently for each direction of travel, increasing it for one and decreasing it for the other, so that there is a net phase shift of $2 \mathrm{~F}$ between the 
counter-propagating beams around the loop. The light is recombined on the beam splitter-detector combination and a fringe shift is observed.

The apparatus can be further improved with a clever scheme for phase modulation analogous to heterodyning as described in Ref. 22. Sensitivity depends on the number of turns of fiber and the current-carrying conductors. Depending on all these parametars, measurements can be made over a large range, bounded by counting many fringes on the high end and the very small fractional fringe counts reached by heterodyning principles on the low end.

An advantage of using the Sagnac interferometer approach is that the phase modulation and detection methods developed for inertial rotation sensors (fiber-optic gyros) are available and less susceptible to common mode polarization effects, and similar in many respects to techniques commonly used In CTR for various interferometer diagnostics.

\section{MAGNETOSTRICTION}

A third approach 23 to measuring magnetic fields involves coating a fiber wth magnetostrictive material and measuring the strain effects with the fiber as one arm of a Mach-Zehnder interferometer. The fiber could also be bonded in some way to a chunk of magnetostrictive material. The Mach-Zehnder Interferometer measures fiber strain regardless of source, and any fleld that can be used to produce a strain in some materlal that can in turn be bonded to an optical fiber is susceptible to measurement in this way. We visualize using discrete magnetostrictive sections distributed along the fiber to produce devices analogous to sine-cosine colls. 
Using the Kerr effect is much more difficult because of the small value of the Kerr constant for silica, $9 \times 10^{-15} \mathrm{cmV}^{-2}$. For a field such as exists in the dense $z$-pinch, $E \sim 25$ to $75 \mathrm{kV}-\mathrm{cm}^{\mathrm{l}}$, the retardation amounts to $1.8 \times 10^{-5}$ to $1.6 \times 10^{-4} \mathrm{rad} \mathrm{cm}^{-1}$ of exposed fiber. An exposed length of 1 to $10 \mathrm{~m}$ would be required to get 1 deg of retardation.

Another possibllity might be to use liquid-core fiber, with a hlgh-Bk 1iquid in the core. Liquids have Kerr constants 2 to 4 orders of wagnitude greater than silica, and a respectable retardation would be avallable for centimeter lengths with a liquid such as nitrobenzene $\left(12.7 \mathrm{rad} \mathrm{cm}^{-1}\right.$ at $75 \mathrm{kV}$ $\mathrm{cm}^{-1}$ ). Liquid-core fibers have been made for years tc avoid some birefringence problems, but are out of vogue at presert. We have contacted some flber manufacturers who have told us such fibers can be made easily on special order, but at a minimum inner diameter of about 25 to 50 m, too large for single-mode operation at visible wavelengths.

VII. FIBER AVAILABILITY

The Low linear birefringence fibers mentioned above are only avallable in Europe. We have purchased $100 \mathrm{~m}$ of such fiber from the University of Southampton for $\sim \$ 600$. Siemons $A G$ has given us $10 \mathrm{~m}$ of their low birefringence fiber in exchange for a promise of reporting our results to them. An American firm, Valtec Corp., has shown an interest in learning the University of Southampton process.

ITT Electro-Optics Division in Roanoke, Va. produces high stress-induced birefringence fibers with elliptical cores that serve as polarization malntaining flbers. They also produce quantities of single-mode fiber for communfcations purposes. We have samples of each, as well as samples of singie-mode fibers from Corning and Fiber Communications, Inc., now part of 
Times and no longer producing single-rode fiber. ITT is a major supplier of fiber to NRL for their research and is also engaged in producing optical fiber couplers that greatly simplify the construction of Interferometers.

\section{WORK IN PROGRESS}

We are building two analog polarimeters such as those described in Refs. 17 and 18 for laboratory work. If a polarfmetric diagnostlc were developed to be used on say, ZT-40, we would expect to do the signal processing with a CAMAC interface to the Prime 400 using modules already in-house. The anaiog polarimeters are designed for a 500-kHz bandwidth and might be marginally suitable for field use, but computer processing the data as described should produce more precision and better frequency response. We also propose to pursue the SAGNAC interferometer approach. We feel it has the most promise in terms of sencttivity, ease of fabrication, and minimization of operator attention in an on-line diagnostlc. Auch of the necessary hardware is not yet available in CTR, however.

There are peripheral technologles that must be mastered in parallel with the main effort. These include the abilitles to handle and splice single-mode fiber and to create optical couplers, and so we have purchased a commercial fustion splicer. We also need to characterize fibers and have been looking at commercial devices for that purpose. To use interferometric methods, we must build phase modulators, and to produce $11 \mathrm{ght}$ properly polarized for our needs, we must make fiber optic devices that are the equivalent of variable wave plates or Babinet-Soleil compensators. 24 
REFERENCES

1. F. P. Kapron, N. F. Bore111, and D. B. Kech, "B1refringence in Dielectric Optical Waveguides," IEEE J. Quantum Electron. 8, 222-225 (1972).

2. A. Simon and R. Ulrich, "Evolution of Polarization Along a Single-Mode F1ber," Appl. Phys. Lett. 31, 5i7-520 (1977).

3. S. R. Norman, D. N. Payne, and M.J. Adams, "Fabrication of Single-Mode Fibers Exhibiting Extremely Low Polarization Birefringence," Elec. Lett. 15, 309-311 (1979).

4. A. Papp and H. Harms, "Magnetooptical Current Transformer. 1: Princtples," Appl. Opt. 19, 3729-3734 (1980).

5. H. Aulich, W. Beck, N. Douklias, H. Harms, A. Papp, and H. Schneider, "Magnetooptical Current Transformer. 2: Components," Appl. Opt. 19, $3735-3740(1980)$.

6. A. Papp and H. Harms, "Polarization Optles of Index-Gradient Optical Waveguide F1bers," App1. Opt. 14, 2406-2411 (1975).

7. A. M. Smith, "Optical Fibers for Current Measurement Applicattons," Optics and Laser Technology (February 1980) 25-29.

8. C. M. Davis, R. E. Einzig, J.A. Bucaro, T. G. Giallorenzi, and V. A. Simmons, "Fiber Optic Sensor System (FOSS) Technology Assessment (Unclassiffed Version)," Prepared for Naval Research Laboratory, 2455 Overlook Ave., S.W., Washington, DC 20375, Dynamic Systems Inc. report No. DSI-TR-80-001.

9. S. E. Miller, E. J. Marcat1111, and T. Li, "Research Toward Optical Fiber Transmission Systems. Part I: The Transmission Medium," Proc. IEEE 61 , $1703-1725$ (1973). 
10. V. Ramaswamy, R. D. Standley, D. Sze, and W. G. French, "Polarization Effects In Short Length, Single Mode Fibers," Bell Sys. Tnch. J., 57, 635-65], (March 1978).

11. V. Ramaswamy, R. H. Stolen, M. D. Divino, and Wipleibel, "Birefringence In Elliptically Clad Borosilicate Single-Mode Fibers," App1. Opt. 18, 4080-4084 (1979).

12. V. Ramaswamy and W. G. French, "Influence of Nrictecular Core on the Polarization Performance of Single Mode Fibres," Elec. Lett. 14, 143-144, (1978).

13. H. Schnelder, H. Harms, A. Papp, and H. Aullch,"Low-Birefringence Single-Mode Optial Fibers: Preparation and Polarization Characteristil:s," App1. Ope. 17, 3035-3037 (1978).

14. W. A. Shurcliff, Polarized Light (Harvard University Press, Cambridge, Mass., 1962).

15. W. Swlndell, Ed., Polarized Light (Dowden, Hutchinson and Ross, Inc., St roudsburg, Pa., 1975).

16. R. M. A. Azzan and N. M. Bashara, Ellipsometry and Polarized Light (North-Holland Publishing Co., New York, N.Y., 1977).

17. D. E. Horne and E. Sawatzky, "Modified Magneto-optic Hysteresigraph," Rev. Sc1. Instrum. 43, 1842-1844 (1972).

18. H. Harms and E. Feldtkeiler, "Automatic Recording of Faraday Rotation and Circular Dichrolsm," Rev. Sci. Instrum. 44, 742-743 (1973).

19. A. M. Smith, "Polarization and Magnetooptic Properties of Single-Mode Optical Fiber," Appl. Opt. 17, 52-56 (1978).

20. S. C. Rashleigh and R. Ulrich, "Magneto-optic Current Sensing with B1refringent Fiber," Appl. Phys. Lett. 34, 768-770 (1979). 
21. R. Ulrich and A. Simon, "Polarization Optics of Twisted Single-Mode Fibers," Appl. Opt. 18, 2241-2251 (1979).

22. H. J. Arditty, Y. Bourbln, M. Papuchon, and C. Puech, "Current Sensor Using State-of-the-Art Fiber-optic Interferometric Techniques," Tech. Dig. of Third Int'1. Conf. on Integrated Optics and Optical Communications, San Franciscs, CalfF. (1981). Paper WL3. (Published by Optical Society of America). IEEE catalog number $81 \mathrm{CH} 1649-3$.

23. J. Jarzyski, J. H. Cole, J. A. Bucaro, and C. M. Davis, Jr., "Magnetic Field Sensitivity of an Optical Fiber with Magnetostrictive Jacket," App 1. Opt. 19, 3746-3748 (1980).

24. H. C. Lefevre, "Single-mode Fibre Fractional Wave Devices and Polarisation Controllers," Eles. Lett. 16, 778-78- (1980). 


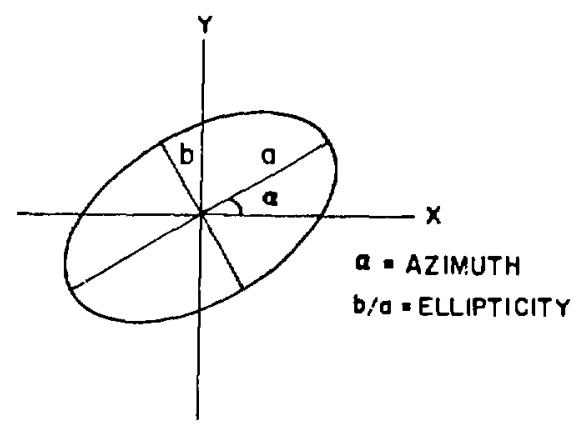

Fig. 1. General polarizatior ellipse defining azimuth and ellipticity.
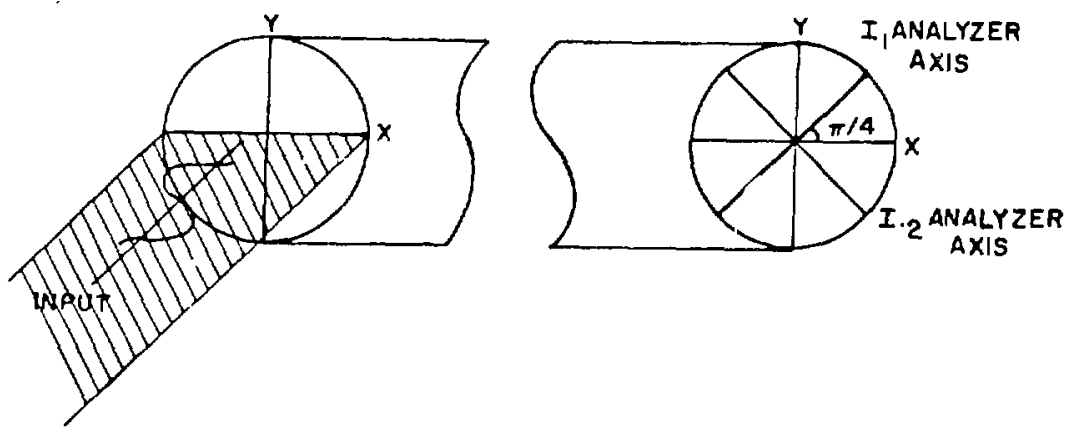

Fig. 2. Coordinates for measuring circular birefringence in the presence of linear birefrirgence. Input polarization is parallel to one of the (local) fiber axes. The two orthogonal output analyzers are at $\pm \pi / 4$ radians to the (local) fiber axes.

FIGURE 3

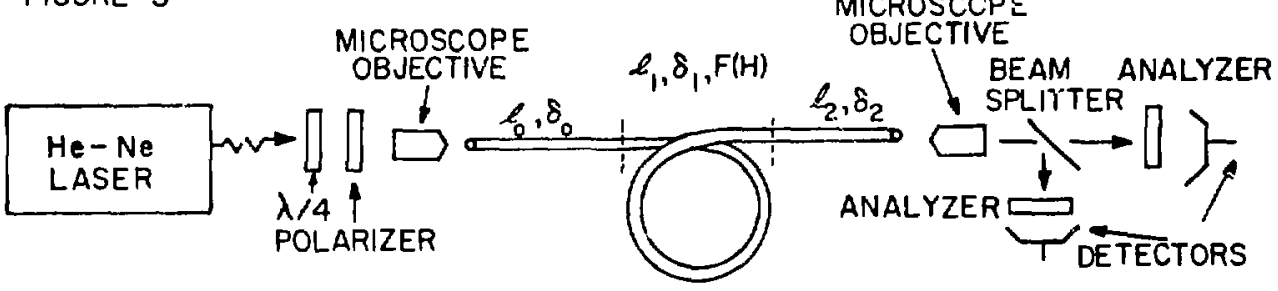

OPTICAL FIBER

Fig. 3. Schematic of apparatus for fiber polarization measurement. See text for definitions of symbols. 


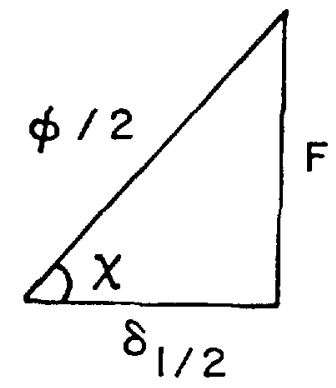

Fig. 4. Definition of $x$ and $\phi / 2$ in terms of Faraday rotation angle $F$ and linear retardation phase angle $\delta_{l}$ in measurement section.

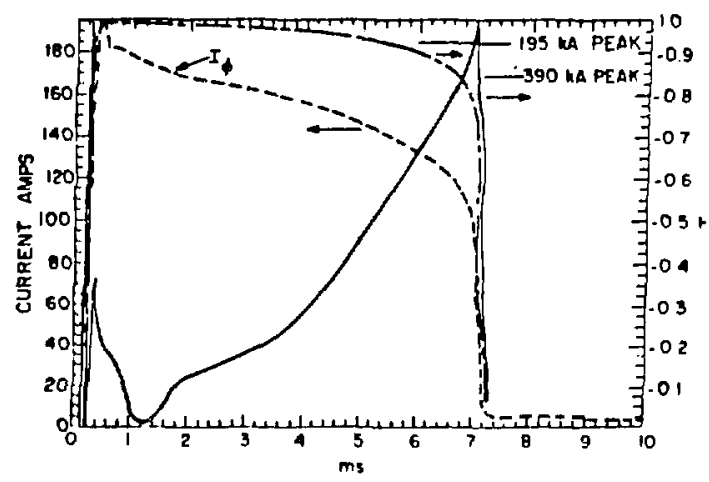

Fig. 6. Convolution of typical ZT-40 $I_{\phi}$ current waveform with Fig. 5 for two different peak currents, showing time response of normalized intensity polarization analyzer.

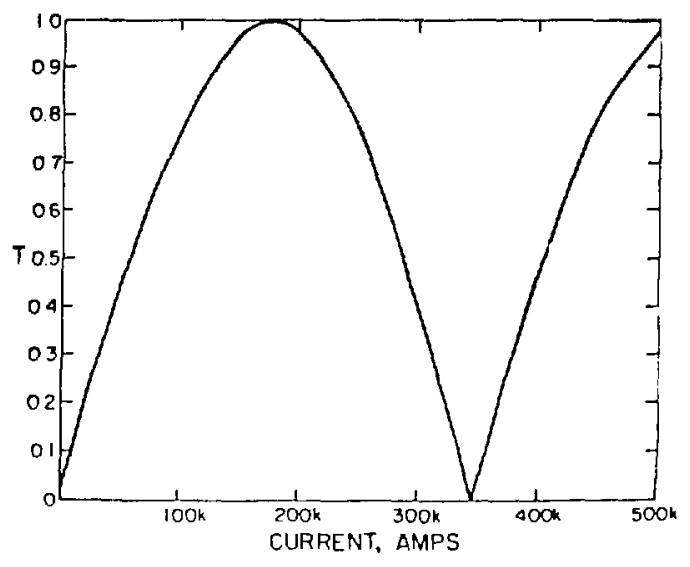

toucth

Fig. 5. Plot of the normalized intensity response of polarization analyzers vs current for singleloop silica fiber wound on $\mathrm{ZT}-40$.

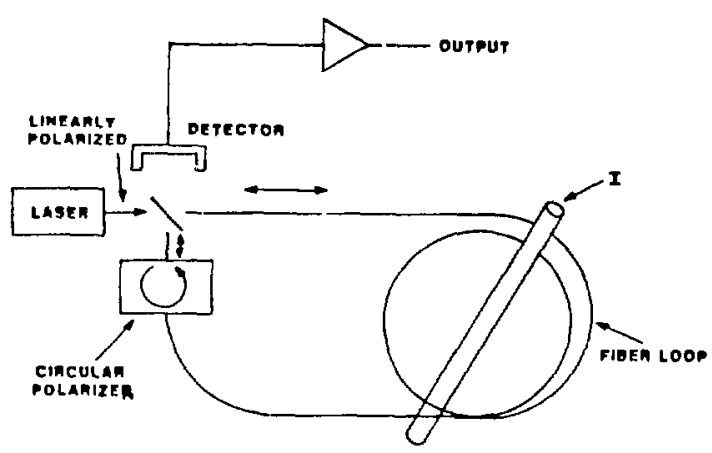

Fig. 7. Conceptual arrangement of Sagnac interferometer for greater sensitivity current sensing. 


\section{APPENDIX}

\section{LOCAIION OF THE FAST AND SLOW AXES OF FIBER TRANSMISSION}

A setup simfliar to Figs, 2 and 3 is used, so that the linearly polarized light is incident on the fiber end at a variable angle $\phi$ to an arbitrary reference direction, and so that the analyzers also can be rotated to a varlable angle $\psi$ to some reference direction. Now the degree of linear polarization $\mathrm{T}$,

$$
T=\frac{I_{1}-I_{2}}{I_{1}+I_{2}},
$$

is measured after first rotating the analyzer axes to maximize $I_{1}$ and minimize $I_{2}$. The angle $\phi$ at which $\mathrm{T}$ is maximized correspouds to the fast or slow axis, and $\phi \pm \pi / 2$ is the other. The analyzer axes at $\mathrm{T}_{\max }$ also correspond to the fast and slow axes. Th1s techalque does not determine which $1 \mathrm{~s}$ which, nor does 1 t matter for our purposes. For inore information see Ref. 6 . 